\title{
Demand on Request and Performance Evaluation in Video MAC Protocol (VMP) for EPON based Network
}

\author{
T.Janarthan \\ M.E Communication \\ Systems/ECE \\ GKM College of Engg and \\ Tech, Chennai-63, India
}

\author{
N.Archana \\ Asst Professor/ECE \\ GKM College of Engg and \\ Tech, Chennai-63, India
}

\author{
K.O.Joseph, PhD. \\ Professor/ECE \\ GKM College of Engg and \\ Tech, Chennai-63, India
}

\begin{abstract}
Video MAC Protocol (VMP) in EPON based access networks. In triple-play (Data, Voice, and Video) service scenarios for such EPON based access networks, Optical network technologies are expected to converge in the near future, since the fiber networks hold great promise as future broadband communication. Video traffic will likely dominate in triple-play service scenarios for each fiber networks. It is proposed to introduce and analyze the Video MAC Protocol (VMP) to efficiently deliver pre-recorded video downstream to consumers over a fiber network in presence of voice and data upstream and downstream traffic. The VMP consists of three main components: (i) Frame Fragmentation and Hierarchical frame Aggregation, (ii) Multi-Polling Channel Access, and (iii) Prefetching for VBR Video Streams. By introducing VMP we are going to analyze the throughputdelay performance for all three traffic types: Voice Traffic, Video Traffic, and Data Traffic. In this paper we are evaluating a demand on request and performance evaluation of VMP. Video streaming according to the user requirement and encoded in three quality types of Video for low, medium and high quality users and the most common performance metrics are delay and throughput are compared with existing Mac protocol.
\end{abstract}

\section{General Terms}

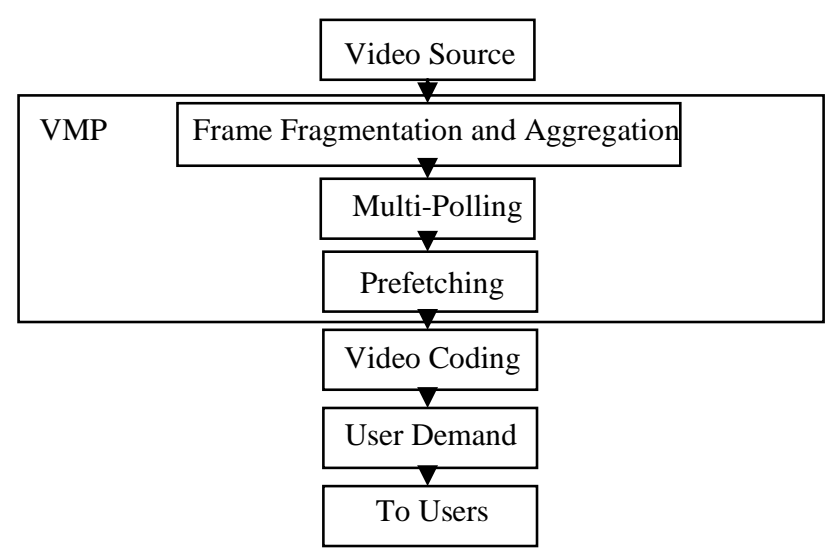

Fig: 1, General Terms of VMP

The evaluation of Video MAC Protocol according to the flow diagram the VMP was deals with three main novels (i) Frame
Fragmentation, (ii) Multi-Polling, and (iii) Prefetching. The novels was studied and implemented according the user demand the service will provided. This terms are the general terms in video mac protocol (VMP) shon in fig-1.

\section{Keywords}

Video MAC Protocol, Video on Demand, EPON, Prefetching, Video Streaming, Video Frame, MAC Protocol, Multi Polling.

\section{INTRODUCTION}

In response to the steadily increasing demand for bandwidth and networking services for residential users as well as enterprise customers, passive optical networks(PONs) have emerged as a promising access technology that offers flexibility, broad area coverage and cost-effective sharing of the expensive optical links compared with the conventional point-to-point (P2P) transport solutions. A Passive Optical Network (PON) is a single, shared optical fiber that uses inexpensive optical splitters to divide the single fiber into separate strands feeding individual subscribers. PONS is called "passive" because, other than at the central office and subscriber endpoints, there are no active electronics within the access network. it was shown that PONs are also more energy efficient than fiber-to-the-home (FTTH) network technologies such as point-to-point and active optical access networks. A key requirement for providing flexible networked video services is to deliver the video frames in a timely manner so that the receiver can continuously play back the video. This timely video frame delivery is made challenging by the highly varying (bursty) video traffic bit rates produced by the efficient video coding standards, especially the H.264 Scalable Video Coding (SVC) standard. To provide quad-play services (Data, Voice, Video, and Mobility) on the same network infrastructure, broadband solutions by deploying PONs and capitalizing on the respective strengths of optical technologies. To provide quad-play services (i.e., voice, video, data, and mobility) on the same network infrastructure, Fiber networks hold great promise as future-proof broadband solutions by deploying PONs and capitalizing on the respective strengths in optical fiber as an analog transmission medium between the central office (CO).

\section{RELATED WORK}

\subsection{Overview of VMP}

Video MAC Protocol (VMP) to efficiently deliver prerecorded video downstream to consumers over a EPON network in the presence of voice and data upstream and downstream traffic. VMP consists of three main novel components: (i) frame fragmentation in conjunction with 
hierarchical frame aggregation for efficient MAC frame transport over the integrated optical network, (ii) multi-polling medium access control for upstream voice and data packets and acknowledgements for downstream video packets, and (iii) prefetching of video frames over the optical network in conjunction with reservation/contention-based medium access.

\subsection{Frame Fragmentation and Aggregation}

Hierarchical (tow-level) MAC frame aggregation has been studied for an EPON. Furthermore, hierarchical frame aggregation for a EPON network has been examined. Noting that all aggregated frames need to be retransmission using a specific acknowledgement technique has been proposed. The proposed fragmentation strategy retransmits only the corrupted fragments. The fragment size plays a key role for efficient fragmentation and for the fragment size settings in our study. Building on these existing techniques, we introduce hierarchical (two-level) frame aggregation with frame fragmentation in this article and study this novel approach in the context of a EPON network.

\subsection{Channel Access Control}

MAC enhancement techniques for EPON with reservations. Frame aggregation, such as multi-user polling controlled channel access (MCCA), which has been introduced in for an isolated next-generation GPON. MCCA divides the channel access time into two periods: (i) non-polling period and (ii) polling period [11]. The access point (AP) gathers the channel access requests of stations (STAs) and broadcasts a multipolling packet, which indicates the polling schedule of each STA [14]. We extend the multi-polling strategy to an integrated FiWi network.

\subsection{Video Traffic Scheduling}

Video transmission over optical access networks has been investigated relatively little to date [9]. Video delivery over hybrid fiber-coax access networks with a focus on traffic prediction and the DOCSIS medium access control and physical layer specifications is studied. Techniques based on multi- and broadcast that periodically transmit segments of popular videos in optical access networks are studied in. In the recent studies periodic transmission schemes are (i) extended with patching mechanisms that allow a receiver to quickly start video playback from a patch stream while storing the next video segments from the periodic transmissions, and (ii) refined with a range of performance enhancing mechanisms, such as downloading popular movies during night hours. These existing studies have in common that they do not consider the variable frame sizes of rate-distortion (RD)-efficient video codec's; instead, they approximate (or bound) the video bit rate as a constant bit rate. In contrast, we consider the highly variable bit rates of H.264 SVC encoded video and examine video frame scheduling mechanisms that efficiently deliver the variable-sized video frames for timely playback.

\subsection{Ethernet Passive Optical Network}

In recent years the telecommunications backbone has experienced substantial growth however, little has changed in the access network. The tremendous growth of Internet traffic has accentuated the aggravating lag of access network capacity [3]-[4]. The "last mile" still remains the bottleneck between high-capacity Local Area Networks (LANs) and the backbone network. The most widely deployed "broadband" solutions today are Digital Subscriber Line (DSL) and cable modem (CM) networks. Although they are an improvement compared to $56 \mathrm{Kbps}$ dial-up lines, they are unable to provide enough bandwidth for emerging services such as Video-OnDemand (VoD) interactive gaming or two-way video conferencing [1]-[2]. A new technology is required one that is inexpensive, simple, scalable, and capable of delivering bundled voice, data and video services to an end-user over a single network. Ethernet Passive Optical Networks (EPONs), which represent the convergence of low-cost Ethernet equipment and low-cost fiber infrastructure, appear to be the best candidate for the next-generation access network.

\subsubsection{Physical Layer}

At the physical layer both standards adopted the same wavelength plan $(1310 / 1490 \mathrm{~nm}$ wavelength band for the upstream/downstream direction, respectively) and transceivers with similar characteristics [3]-[4]. EPON can provide a net $1000 \mathrm{~Gb} / \mathrm{s}$ data rate with respect to the 1250 $\mathrm{Gb} / \mathrm{s}$ line rate due to the adoption of $8 \mathrm{~b} / 10 \mathrm{~b}$ encoding, chosen to simplify the receiver design.

\subsubsection{Transmission Convergence}

The purpose of the TC layer is to reconcile the point-to-multipoint (P2MP) shared physical link of the PON tree with the higher layer protocols, which have been designed for use in $\mathrm{P} 2 \mathrm{P}$ links. It provides for de-multiplexing frames from the MAC layer based on a unique identifier per ONU indicated inside the frame header. Frames are filtered based on this identifier and classified into separate queues emulating discrete logical P2P links between the OLT and the multiple ONUs of the different customer interfaces. This allows reaping the economic advantages of sharing the expensive physical resources, while keeping the appearance of a bunch of dedicated links with ordinary protocols above the TC protocol.

\subsubsection{MAC Layer}

Both standards allow for the implementation of a systemspecific Dynamic Bandwidth Allocation (DBA) mechanism: ONUs may dynamically request transport capacity by sending their queue length reports and the OLT schedules the time slots for ONUs based on their requests thus, adapting to traffic fluctuations. In both cases only the type of messages that should be exchanged during operation are defined, in order to guarantee interoperability, without specifying exact algorithms that can be employed especially for bandwidth allocation [3]-[13]. This is left open to the vendors and network providers to handle according to their specific requirements. In the EPON case, the multi-point control protocol (MPCP) is used to arbitrate the upstream access. MPCP uses two types of messages (encapsulated in Ethernet frames) during normal operation for arbitration of packet transmissions: the REPORT message used by an ONU to report the status of its queues to the OLT (up to eight reported in a single message) and the GATE messages issued by the OLT and indicating to the ONUs when and for how long they are allowed to transmit in the upstream channel [3]. Each GATE message can support up to four transmission grants targeting individual service entities within the same ONU (i.e. data queues). In the upstream, the granted ONU transmits (possibly) multiple Ethernet frames as many integral packets as can fit into the allocated transmission slot, since 
fragmentation is not allowed from one or more queues proceeded by the indispensable physical layer overhead. It may also transmit report messages in order to request additional grants. In EPONs, the traffic streams arriving at the ONUs from the customer premises are kept in queues [3]. In compliance to the $802.1 \mathrm{p}$ prioritization scheme, it is possible to inject the traffic in up to eight logically separate, possibly prioritized, queues holding Ethernet frames, with different QoS requirements, to allow for the enforcement of different service mechanisms.

\subsubsection{SVC}

In general, a video bit stream is called scalable when parts of the stream can be removed in a way that the resulting substream forms another valid bit stream for some target decoder, and the sub-stream represents the source content with a reconstruction quality that is less than that of the complete original bit stream but is high when considering the lower quantity of remaining data. Bit streams that do not provide this property are referred to as single-layer bit streams. The usual modes of scalability are temporal, spatial, and quality scalability.

Spatial scalability and temporal scalability describe cases in which subsets of the bit stream represent the source content with a reduced picture size (spatial resolution) and frame rate (temporal resolution), respectively. With quality scalability, the sub-stream provides the same spatial-temporal resolution as the complete bit stream, but with a lower fidelity-where fidelity is often informally referred to as signal-to-noise ratio (SNR). The basic SVC design can be classified as layered video codec. In general, both the coder structure and the coding efficiency depend on the type of scalability that is required by an application. As an important feature of the SVC design, most components of H.264/MPEG4-AVC are reused as specified in the standard. The base layer of an SVC bit stream is coded in compliance with H.264/MPEG4-AVC, and each standard compliant H.264/MPEG4-AVC decoder is capable of decoding this base layer when it is provided with an SVC bit stream.

\subsubsection{Video-On-Demand}

Video-on-demand (VoD) is gaining popularity as a very important application in the entertainment domain. The term video-on-demand is sometimes used to describe pay per view (PPV) or near video on demand (N-VOD). PPV is usually limited to a small number of channels and N-VOD broadcasts particular movies at staggered intervals. However, either PPV or N-VOD provides any interactive functionality to the user. True video-on-demand supports full interactivity, therefore it has the potential to start immediately when requested and it is able to provide full VCR functionality. Users are able to fast forward, pause, rewind and play the video just like a VCR. Hence true video-on-demand involves the transmission of a dedicated video stream for each and every request [15]. A VoD system can be designed with three major network configurations: centralized, networked and distributed. In a centralized system there is one central server that stores all the video content. All the clients are connected to this server to satisfy their requests.

In networked system architecture, multiple video servers are distributed throughout the network. Each video server controls and manages a subset of the video content and is responsible for a small set of client requests. In a distributed configuration there is one central server that stores all the content with smaller servers located near the network edges that are used to store high demand content. When a client requests that a particular video be played, the video server responsible for the request reserves sufficient processing capacity and network bandwidth for the video stream to guarantee continuous playback of the video. Transmission of video streams requires high network bandwidth and this can be very expensive, especially in the core section of the network. Network bandwidth is therefore considered to be the most expensive resource in a VoD system and is a major hurdle in providing an affordable true video-on-demand service.

High transmission cost is a continuing problem hindering the widespread proliferation of VoD. Therefore a critical part of a VoD system design is to optimize network bandwidth. Extensive research has been conducted to solve the bandwidth problem and design cost effective, reliable, efficient and robust video-on-demand systems. However these techniques have significant overheads and their efficiency decreases dramatically as the level of interaction in the system increases. These limitations mean that the techniques are not suitable for commercial use. This paper proposes a VoD model that reduces the bandwidth requirement of the system and has the following three advantages: 1) there are 110 overheads associated with this model; 2) the level of interaction available to the user is not affected and, 3) there is no reduction in the efficiency of the system when the level of interaction increases. Simulation results have shown that bandwidth savings of up to $40 \%$ can be achieved.

\subsubsection{Video Streaming}

Video streaming over networks has received a great deal of attention over past two decades, see for instance. Our study relates to the following three main lines of research: $(i)$ research on the rate-distortion (RD) characteristics of encoded video, (ii) research on the analysis and modeling of video traffic, and (iii) research on video traffic management mechanisms. Research on the RD characteristics of encoded video examines the relationship between the (mean) bit rate and the video quality (and encoder quantization scale), this area of research. The two main approaches that have been employed in RD research are analytical modeling and empirical modeling. Analytical modeling, such as pursued in attempts to derive mathematical formulas for the RD behavior in terms of the statistics of the source video and the properties of the encoding mechanism empirical modeling, as studied in strives to approximate the RD curve by interpolating between a set of sample points.

A unified RD analysis framework, which builds on an analysis of the percentage of zeros in the transformed video 
frames in conjunction with rate curve modeling is developed in the modeled RD characteristics are typically used to control the mean bit rate of video encoders and can also be used for allocating mean bit rates to video streams for network transport.

Our study differs from this literature on the RD characteristics in that we examine the relationship between the variability of the bit rate on the one hand, and the video quality (and quantization scale) on the other hand. In other words, the existing RD studies have focused on the first order statistic of the video traffic, whereas our focus is on the second order statistic (which we study in an empirical manner). The statistical analysis of video traffic and the development of video traffic models has also received significant interest.

\section{SYSTEM DESIGN}

\subsection{Process Flow}

Step 1: Encode the Video Acquisition into three different schemes (Quality 1, Quality 2, Quality 3).

Step 2: Initiate the authentication and Routing procedures

Step 3: User Login Database is updated with the additional information of user bandwidth efficiency for receiving the requested broadcast.

Step 4: Admin Server checks for the user validation and other mandatory reports like login failures, password changes etc..

Step 5: The Bandwidth efficiency of users will be known to receiver, with that it will control the user routing Channel.

Step 6: User will receive Quality reception according to their Bandwidth requirements.

The process flow shows that how the video is given to the users according to their requirements in fig- 2 .

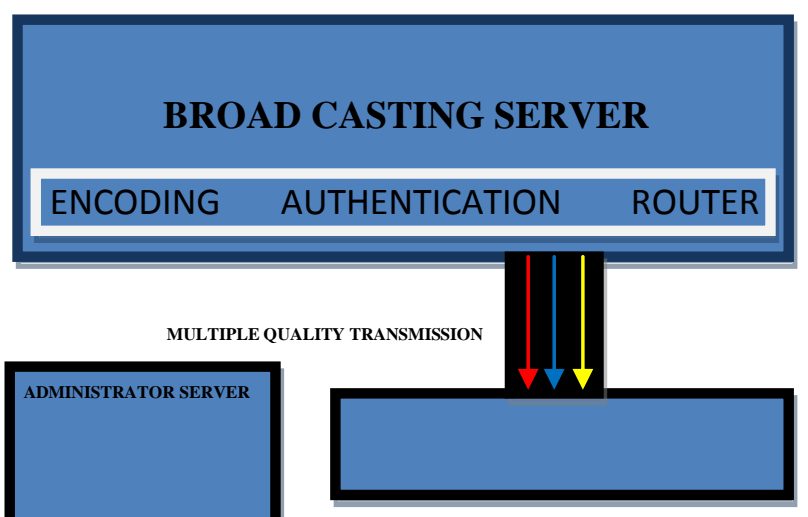

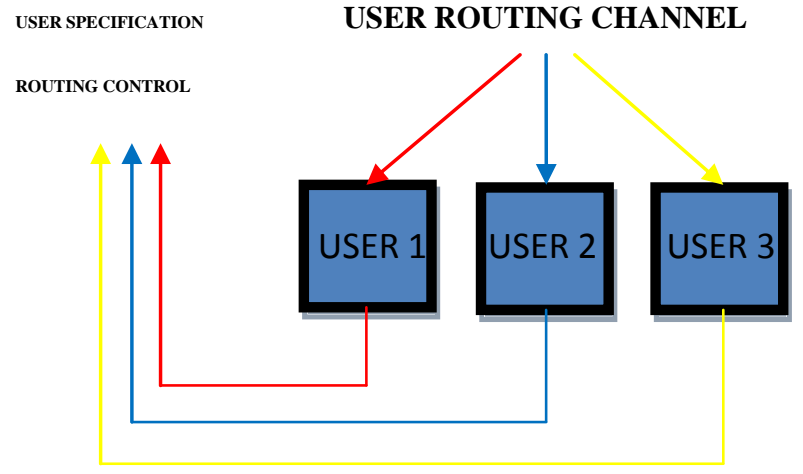

Fig:2, Process Flow

\subsection{Data Flow}

MAC protocol mechanisms are employed across the optical networks to improve down and upstream packet delivery (frame fragmentation and aggregation), upstream packet and acknowledgement delivery (multi-polling), and video streaming in the downstream direction (prefetching) are shown in fig-3.

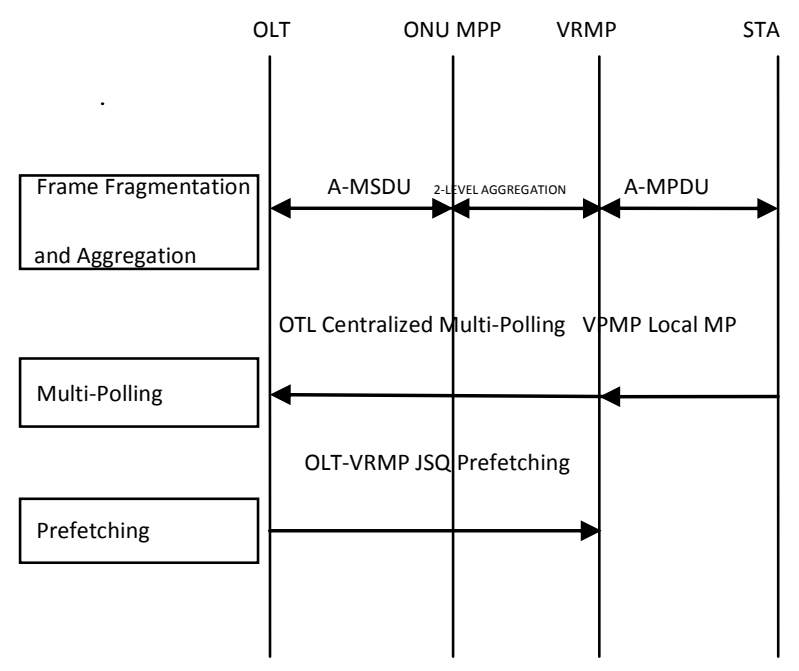

Fig: 3, Data Flow Diagram of VMP 


\section{SIMULATION RESULT}

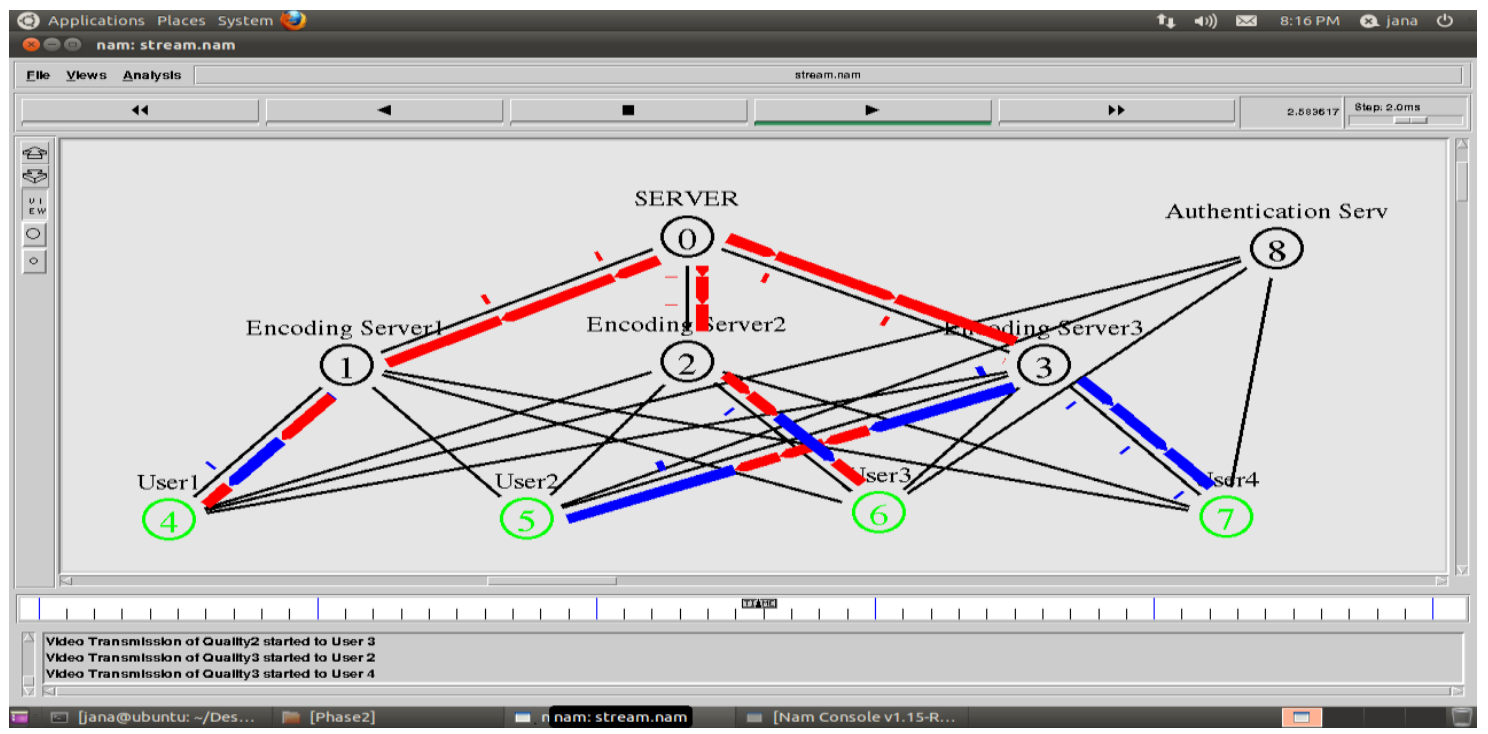

Fig: 4, Service Provided to Users According to their Request

In simulation, The video is received by the client using MAC and VMP protocol. The Network Simulator 2 (Ns2) and $\mathrm{C}++$ and is simulation modules for the different network components and protocol aspects that VMP evaluation compare with MAC protocol is simulated [16].

The Fig: 4, shows that the User-1 sends the request to authentication server and it was approved and the User-2 sending request to authentication server and waiting for approval and the Data, Voice and Video (Triple- Play) using VMP was delivered to the client from the server according to their request. In Fig: 3, It shows the three encoding servers that which provides the different quality of service to the users.

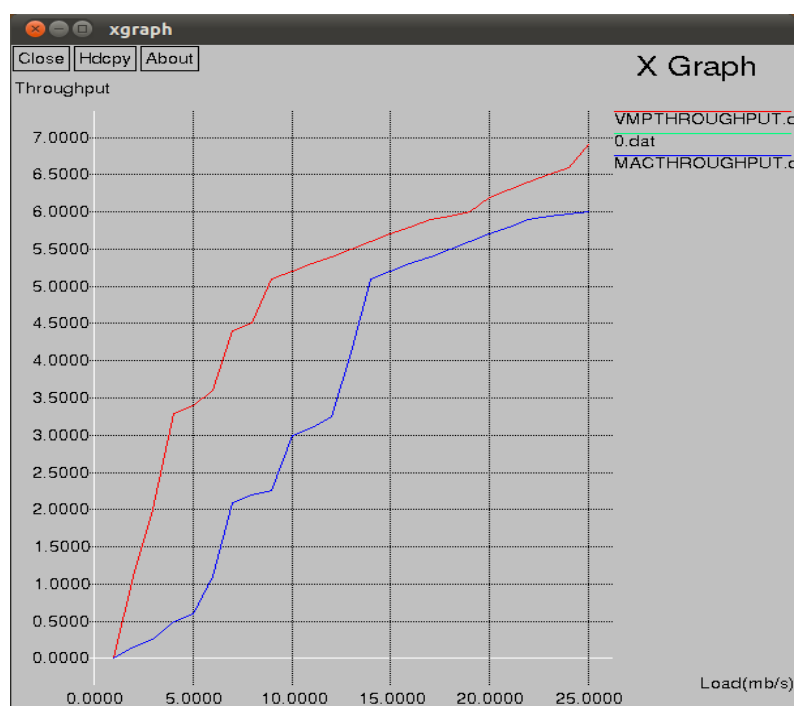

Fig: 5, Throughput performance of VMP and MAC
The above shown fig- 5 shows that the difference between the MAC and VMP protocol and we found that VMP have high efficiency, throughput, delay and energy is increased in VMP as well as the below shown fig- 6 the VMP as minimum delay compare to MAC protocol.

This fig-5 \& 6 shows the performance evaluation of Video MAC Protocol and the existing protocol MAC. The VMP it as high performance and throughput compare to MAC protocol in fig-5. The VMP it minimum delay compare to MAC protocol is shown in fig- 6 . This result shows the Video MAC Protocol is much suitable then the MAC protocol and it can used to provide according to the user demand which according to their bandwidth.

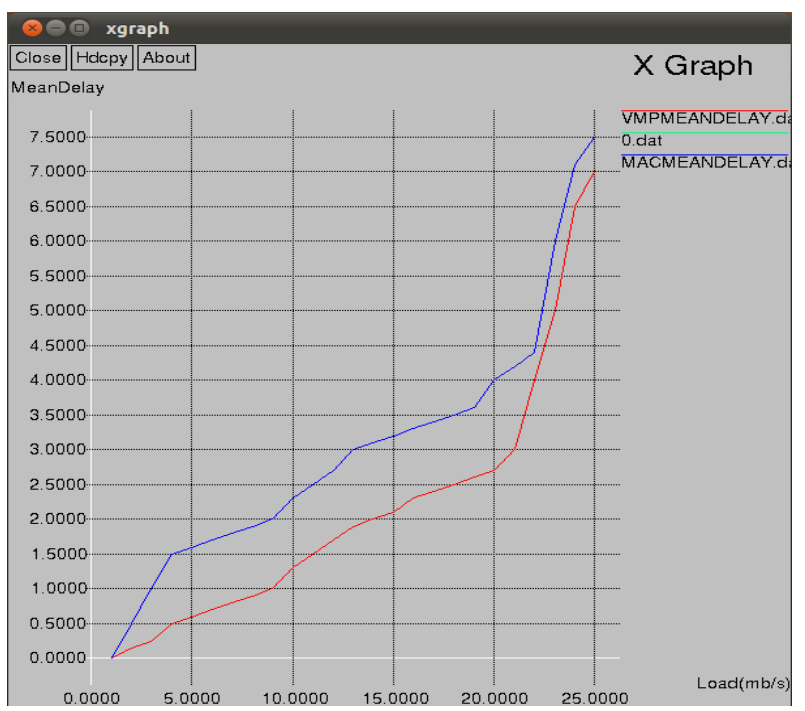

Fig: 6, Mean Delay performance of VMP and MAC 


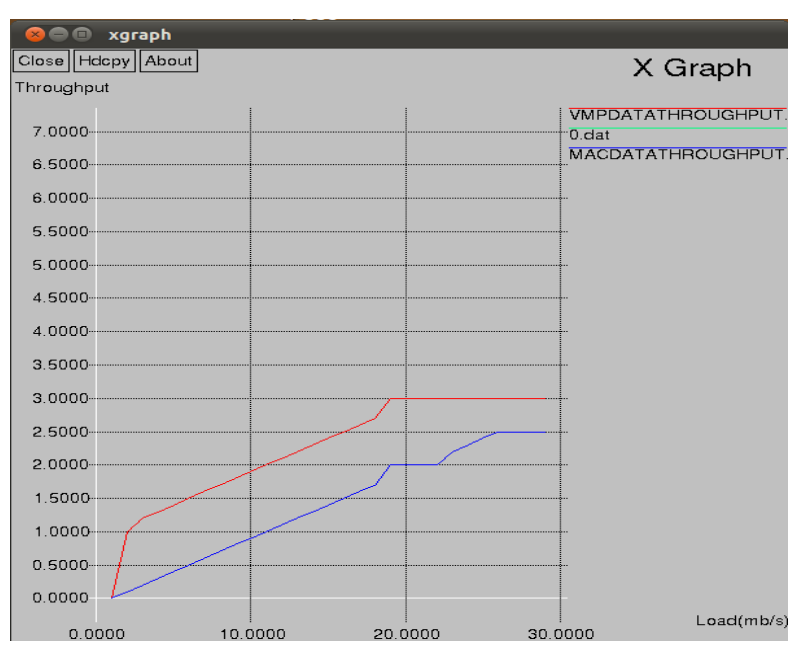

Fig: 7,Data traffic throughput performance of VMP and MAC

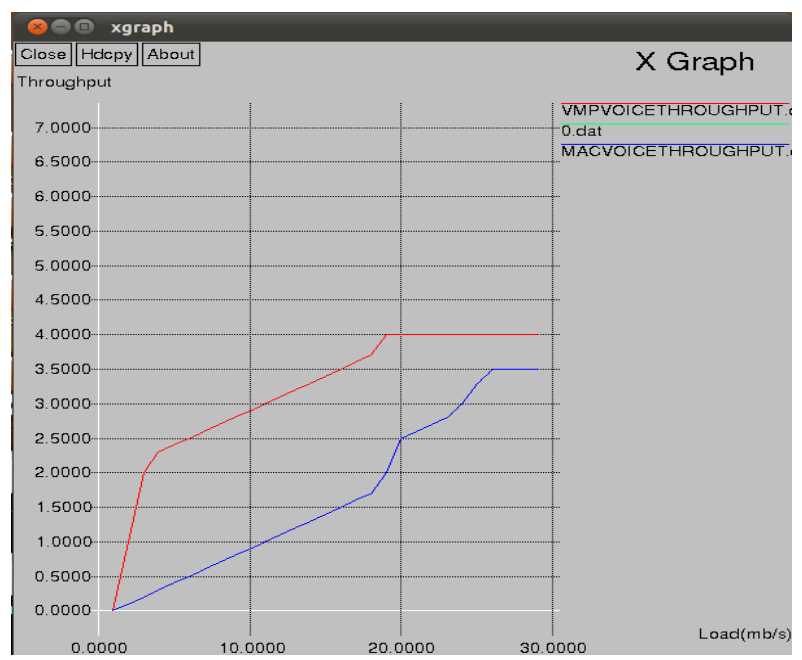

Fig:9,Voice traffic throughput performance of VMP and MAC

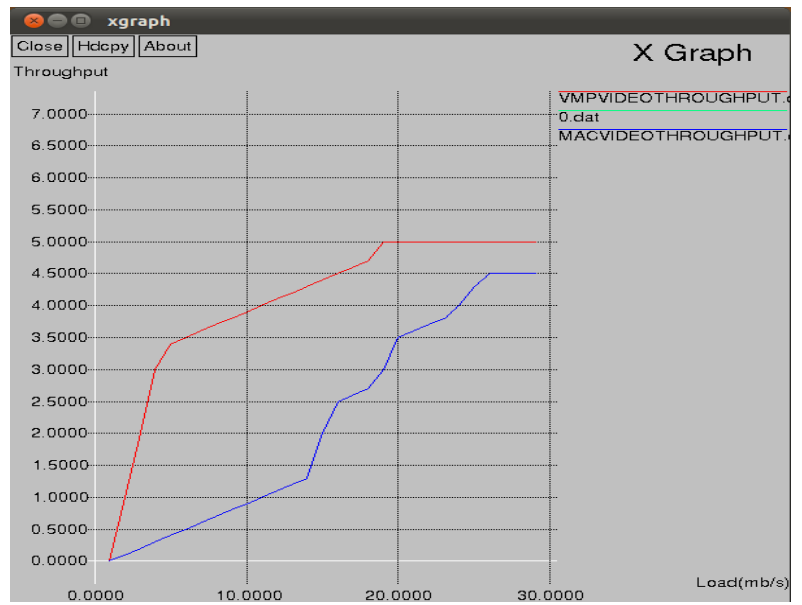

Fig:11,Video traffic throughput performance of VMP and MAC

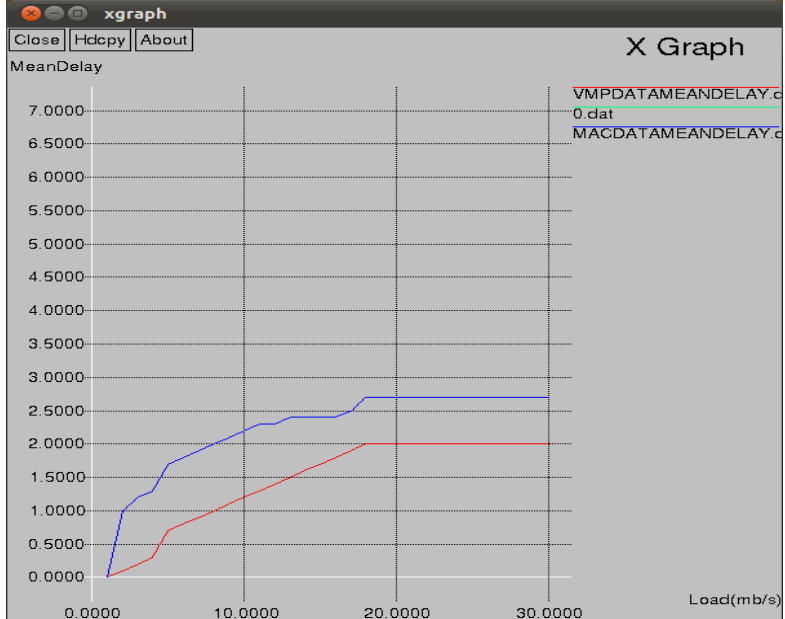

Fig: 8,Data traffic mean delay performance of VMP and MAC

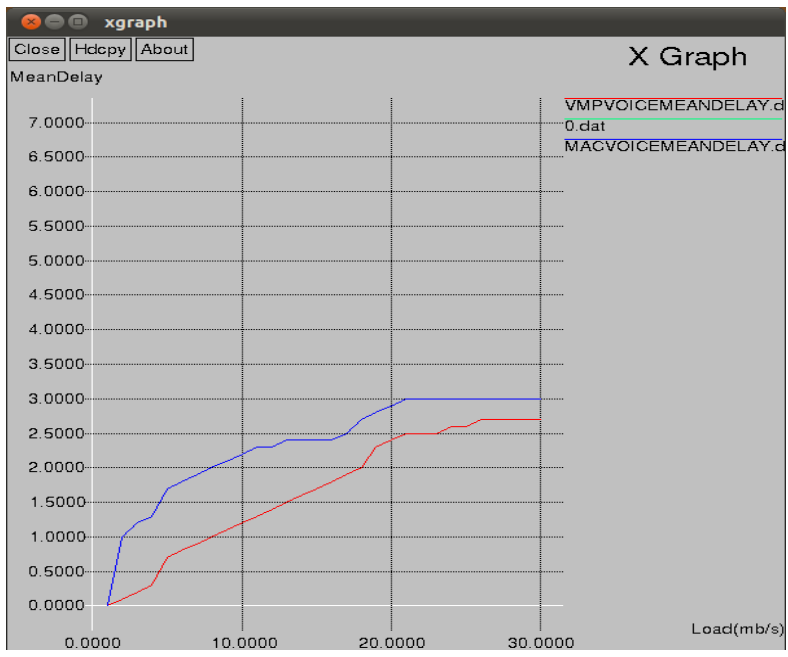

Fig: 10,Voice traffic mean delay performance of VMP and MAC

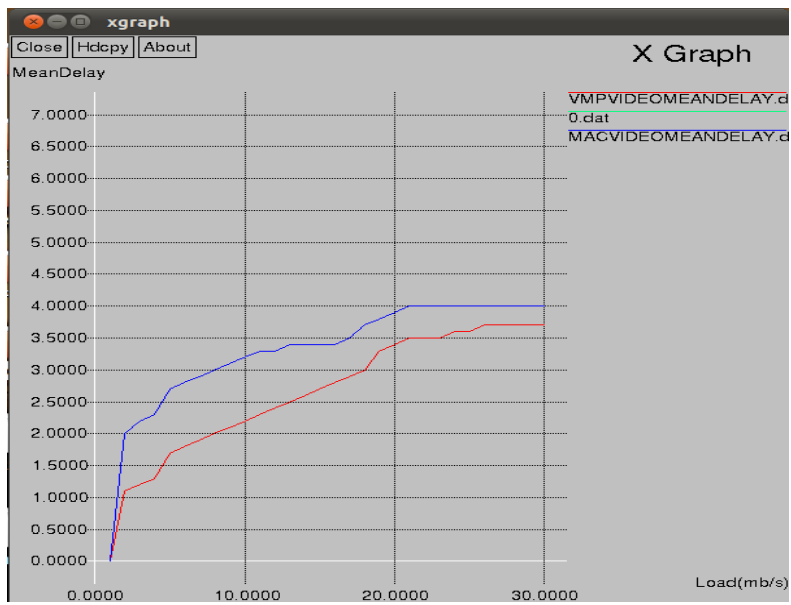

Fig: 12,Video traffic delay performance of VMP and MAC 


\subsection{Demand-On-Request}

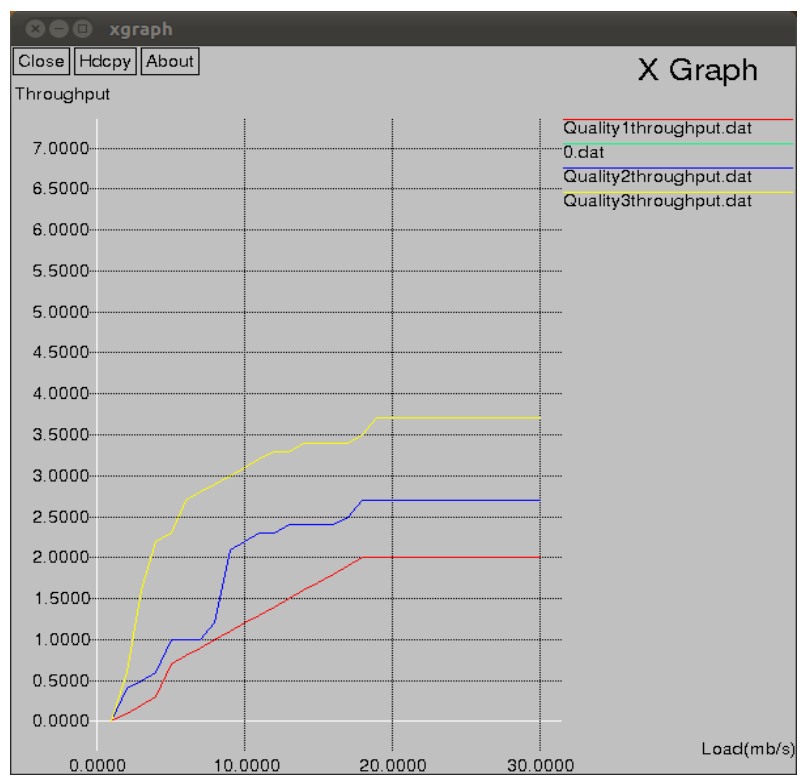

Fig: 13, Throughput of the different quality of video

\section{VMP Performance for Triple-Play}

In fig-7, 8, 9, 10, $11 \& 12$, we plot the throughput and delay for video traffic and data traffic when VMP with prefetching (without channel probing) is employed in the triple-play scenario. We observe from fig-11 \& 12 that video traffic benefits significantly from the combination of joint frame fragmentation and hierarchical frame aggregation combined with multi-polling and prefetching. Similarly, we observe from fig-7 \& 8 that data traffic benefits from the performance enhancing mechanisms in VMP. (The results for voice traffic, which are not plotted, are similar.)

Aside from benefiting from the frame fragmentation and frame aggregation as well as the multi-polling, the downstream voice and data traffic benefit from the more efficient video transport with prefetching. Data traffic is not starved despite the strategy of the JSQ prefetching to transmit as many future frames as fit fully into the total bandwidth and the station bandwidth. There is typically some unused bandwidth remaining after the JSQ policy has removed all streams from consideration because all frames have been transmitted for a stream or none of the next frames of the streams fits fully into the remaining bandwidth. Voice and data traffic, which have much lower traffic rates than the video streams, fill up the remaining bandwidth.

\section{CONCLUSION}

The efficient Medium Access Control (MAC) mechanisms for EPON based network and compared with the Video MAC Protocol (VMP) is discussed. Video MAC Protocol (VMP) is to efficiently deliver pre-recorded video downstream to consumers over a EPON network in the presence of voice and data upstream and downstream traffic pre-recorded video traffic in the downstream direction. It is found that the

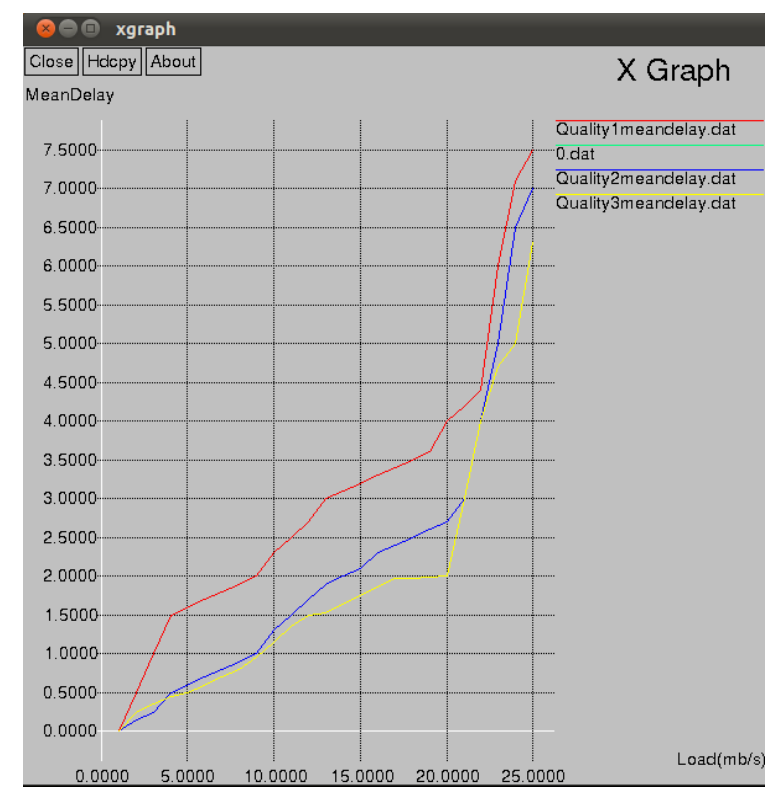

Fig: 14, Mean Delay of the different quality of video

combination of (i) joint frame fragmentation and hierarchical frame aggregation, (ii) multi-polling, and (iii) Pre-fetching in conjunction with EPON reservation/contention-based MAC efficiently supports triple-play (data, voice, and video) traffic in EPON networks. VMAC is compared with the MAC and its mean throughput and mean delay is simulated and its result shown below in fig-5, 6, 7, 8, 9, 10, $11 \& 12$. The overall throughput and delay performance of VMP and MAC shown in fig-5 and 6. The Data traffic throughput and delay performance of VMP and MAC shown in fig-7 and 8. The Voice traffic throughput and delay performance of VMP and MAC shown in fig-9 and 10. The Video traffic throughput and delay performance of VMP and MAC shown in fig-11 and 12. We got the better performance of VMP compared to MAC protocol. the throughput of the different quality of video to the different users is shown in fig-13. The mean delay of the different quality of video to the different users was obtained in fig-14. Demand on request video streaming according to the user requirement we can encode the video in three quality types for low, medium and high quality users was obtained.

\section{FUTURE WORK}

For future improvements, we can implement this protocol in FIWI and LRPON based network. In this we will come up with better performance in throughput, delay and energy consumption. 


\section{REFERENCES}

[1] J. Choi, M. Yoo, and B. Mukherjee, "Efficient video-ondemand streaming for broadband access networks," IEEE/OSA Journal on Optical Communications and Networking, vol. 2, no. 1, pp. 38-50, Jan. 2010.

[2] J. Wang, C. Qiao, Y. Li, and K. Lu, "On guaranteed VoD services in next generation optical access networks," IEEE Journal on Selected Areas in Communications, vol. 28, no. 6, pp. 875-888, Aug. 2010.

[3] G. Kramer, B. Mukherjee, and G. Pesavento, "IPACT: A dynamic protocol for an Ethernet PON (EPON)," IEEE Communications Magazine, vol. 40, no. 2, pp. 74-80, Feb. 2002.

[4] F. Aurzada, M. Scheutzow, M. Herzog, M. Maier, and M. Reisslein, "Delay analysis of Ethernet passive optical networks with gated service," OSA Journal of Optical Networking, vol. 7, no. 1, pp. 25-41, Jan. 2008.

[5] S. Bakiras and V. Li, "Maximizing the number of users in an interactive video-on-demand system," IEEE Trans. Broadcast., vol. 48, no. 4, pp. 281-292, Dec. 2002.

[6] R. Ruby and J. Pan, "Video streaming with PCA and hard vs soft DRP," in Proc., IEEE Globecom, Dec. 2010.

[7] C. Lange and A. Gladisch, "On energy consumption of telecommunication networks-A network operator's view," in Proc., OFC/NFOEC, Workshop on Energy Footprint of ICT, San Diego, CA, USA, Mar. 2009, pp. $1-3$.

[8] G.M.Muntean, P. Perry, and L.Murphy, "A new adaptive multimedia streaming system for all-IP multi-service networks," IEEE Trans. Broadcast., vol. 50, no. 1, pp. 110, Mar. 2004.

[9] N. Ansari, H. Liu, Y. Shi, and H. Zhao, "On modelling MPEG video traffics," IEEE Trans. Broadcast., vol. 48, no. 4, pp. 337-347, Dec.2002.

[10] S. Ou, K. Yang, and H.-H. Chen, "Integrated dynamic bandwidth allocation in converged passive optical networks and IEEE 802.16 networks," IEEE Systems Journal, vol. 4, no. 4,pp.467-476,Dec. 2010.

[11] M. Reisslein and K. W. Ross, "High-performance prefetching protocols for VBR prerecorded video," IEEE Network, vol. 12, no. 6, pp. 46-55, Nov./Dec. 1998.

[12] G. Kramer, B. Mukherjee, and G. Pesavento, "IPACT: A dynamic protocol for an Ethernet PON (EPON)," IEEE Communications Magazine, vol. 40, no. 2, pp. 74-80, Feb. 2002.

[13] P. Wang and W. Zhuang, "A collision-free MAC scheme for multimedia wireless mesh backbone," IEEE Trans. Wireless Communications, vol. 8, no. 7, pp. 3577-3589, Jul. 2009

[14] W.K. Kuo, "Efficient traffic scheduling for real time VBR MPEG video transmission over DOCSIS-based HFC networks," IEEE/OSA Journal of Lightwave Technology, vol. 27, no. 6, pp. 639-654, Mar. 2009.

[15] Santosh Kulkarni, "Bandwidth Efficient Video-ondemand Algorithm (BEVA)" IEEE 2003.

[16] Network Simulator $\quad 2 \quad$ (Ns2) at http://www.isi.edu/nsnam/ns 